\title{
Household Water Resource Monitoring and Leakage Detection
}

\author{
Salman Asif S, Sachin R Doddaguni, Trilok Girish Kamagond, Anala M R, Mamatha T
}

\begin{abstract}
Global environmental situation necessitates water management and conservation. Substantial water wastage can occur by leakage in the water supply system in household areas. Water usage monitoring is imperative in this era of declining water resources.This paper proposes a system to combat the above listed issues using enabling technologies like IoT and cloud services along with machine learning and sensor fusion techniques .In this paper we present a water leakage and monitoring system using cloud computing and IoT. Our proposed water leakage model is based on the idea that there is a relationship between flow values at various places and level of water in the tank. Cloud computing enables remote water usage monitoring and data processing capabilities.A novel method indicating the user to clean their overhead tank is also proposed in this paper.
\end{abstract}

Keywords: cloud computing ,data processing, IoT

\section{INTRODUCTION}

$\mathrm{W}$ ater is a critical component of everyday human life. Due to scarcity of water at global scale its conservation is of utmost importance. Water resource availability in household areas has been declining in the last decade. In a situation of worsening water scarcity problem and higher water demands, water loss management is imperative.The water supplied to households need to be monitored continuously for two reasons: First being the leakage of water anywhere in the house leads to substantial wastage of water resources. Second being the fact that excessive use of water in houses is the major cause of water depletion. Loss of water in households due to leakage in water supply networks is substantial. Water leakage can occur at various places in a household. Leakage is usually because of pipe bursts. Frozen pipes and higher water flow pressure can be the reasons for pipe bursts, such incidents needs to be detected and reported to the user, so that water loss can be minimized. One of the common ways used for monitoring water currently is by the scheduled inspections which involves human intervention.

Revised Manuscript Received on May 05, 2020.

* Correspondence Author

Salman Asif S*, Student Of R.V College of Engineering, Bengluru, Karnataka, India.

Sachin R Doddaguni, Student Of R.V College of Engineering, Bengluru, Karnataka, India.

Trilok Girish Kamagond, Student Of R.V College of Engineering, Bengluru, Karnataka, India.

Anala M R, Student Of R.V College of Engineering, Bengluru, Karnataka, India.

Mamatha T, Assistant Professor, Department of Computer Science \& Engineering at RV College of Engineering, Bengluru, Karnataka, India.

(C) The Authors. Published by Blue Eyes Intelligence Engineering and Sciences Publication (BEIESP). This is an open access article under the CC BY-NC-ND license (http://creativecommons.org/licenses/by-nc-nd/4.0/)
But such maintenance works are time consuming to the worker personnel and is also expensive to client who adopt such measures. Since maintenance is scheduled from time to time, it is possible that the problems may appear much before and go unnoticed. There are already water monitoring systems but they lack real time capability, consume a lot of power and mainly concentrate on the water quality monitoring and not on consumption and leakage detection. In order to avoid human inspection, the solution is to automatically monitor water resources. Hence, the main focus of the work is to monitor the water usage and to some extent water quality through cost effective IoT enabled ideas which give a way for automatic monitoring and avoiding human inspection and also to make the system real time and energy efficient. For this purpose, the proposed system continuously collects data from the sensors and analyses the data using sensor fusion and machine learning to produce desirable outputs regarding the water usage, quality, leakage and also predict the future usage. Consequently, the results can be used to alert the users of their usage, also alert them if any problem is detected in their water system and the quality of water they are using. The system is implemented through the usage of sensors that are available today. These sensors are connected to the microcontroller at each branch of the water system. A considerable amount of work has been done on water monitoring and leakage detection. A smart water monitoring system for smart villages using lora is presented in [1].The system presented made use of the water quality sensors which were connected to the controller and were communicated using M2M Lora which is a very efficient protocol (communication can take place between machines without human intervention) newly developed for long distance communication at a lower rate. An efficient and a low cost water monitoring system exclusively for rivers is discussed in [2, 3]. The system was implemented using the sensors such as temperature, dissolved oxygen, $\mathrm{pH}$, sulphate, nitrate, ammonia, turbidity which were connected to a controller forming a Wireless Sensor Node (WSN) which sends heterogenous data via zigbee and is delivered to the water monitoring control. An IoT enabled overhead tank water quality monitoring system is presented in $[4,5]$. The system used water quality sensors like temperature, $\mathrm{pH}$ etc. Here the sensor nodes were accessed through the PAN type network. A water resource monitoring system is proposed [6] which use water quality sensors .A Raspberry-Pi and a wifi module is used for data processing and data communication respectively. This system cannot be used to transmit data to long distances. Hence, can be used only for short distances. An IoT enabled water quality monitoring system [8] where level was continuously monitored to warn in prior regarding floods through a cloud server with the help of the internet and the information is also sent to the subscribed users .

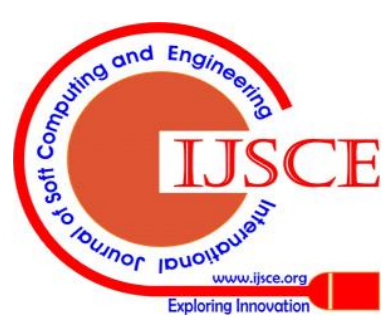




\section{Household Water Resource Monitoring and Leakage Detection}

Social media websites like twitter is also used to alert.

The level was continuously monitored through the use of ultrasonic sensor. It was connected to a microcontroller and an alert was being sent to the cloud server and the twitter handle. [7] discusses a cost effective method to filter dirt in overhead tanks using LDR sensor.LDR sensor responds to variation of light intensity in the overhead tank.

The remaining part of our paper is presented as follows: Section II presents the system architecture. Section III shows the operation of our water system model. Section IV shows the results of the model and next Section $\mathrm{V}$ gives the conclusion and future works.

\section{SYSTEM ARCHITECTURE}

\section{A.water resource monitoring}

The proposed automatic water monitoring system consists of :

1. Ultrasonic sensor, which is used to determine the depth level of water in the tank.

2. Turbidity level is determined by LED and LDR circuit system present inside water tank.

3. Microcontroller unit which is used to collect the sensor data(nodemcu).

4. Flow sensors which are connected to each branch of the water system to monitor flow rate.

The proposed model uses a model built to simulate a household water network. Various factors are considered while building the model. The ultrasonic sensor, LED and LDR circuit system are placed in the tank which are connected to the microcontroller unit. Whereas, the flow sensors are connected to each branch of the water system and have an individual microcontroller at each branch of the water system for collecting data.

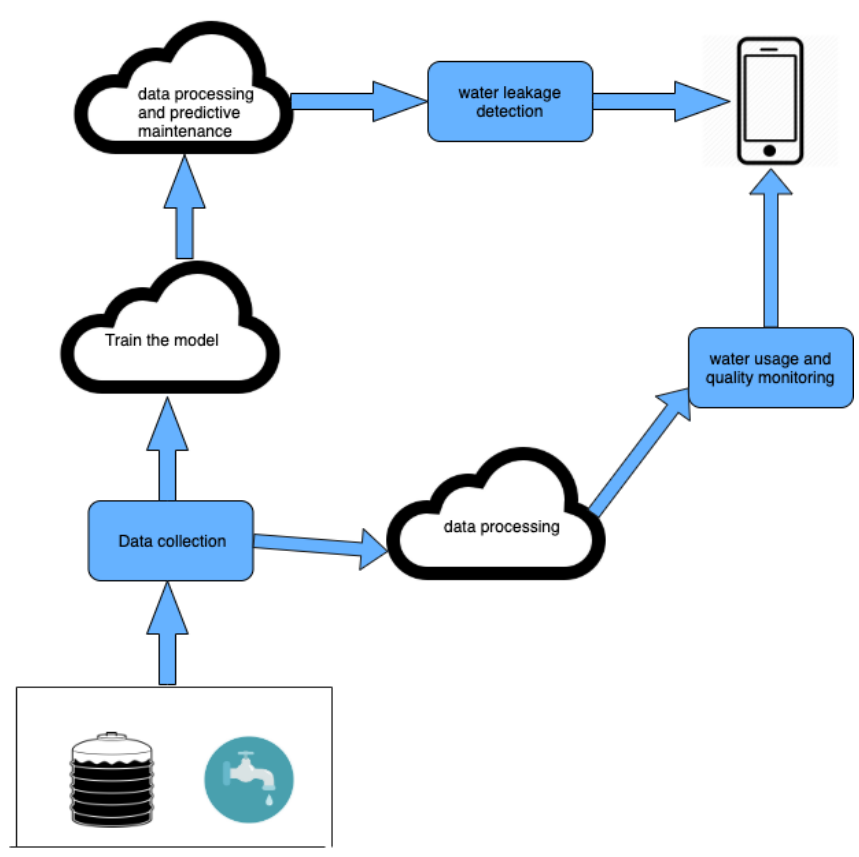

Fig. 1. Block Diagram of Proposed System

The figure1 describes the architecture of the proposed model. Data collected from the sensor is uploaded to cloud. Pre-processing is done on the data followed by training the

\section{Rotripial lal Number: F3393039620/2020@BEIESP

model. The data is also processed to obtain water usage and turbidity levels in the household tank and the details are further displayed on the mobile App.

\section{B. LED and LDR circuit system as turbidity sensor}

The LDR or Light dependent Resistor is a sensor that can be utilized to measure the particulate matter or cloudiness of water in the tank. The circuit system consists of placing LED or Light Emitting Diode directly and in line opposite to LDR. LDR is constituted of high resistance semiconductor material and inverse property of resistance with increase in light intensity is used. As the intensity of light falling on the LDR sensor increases, the material is acquiring more photons and the energy of photons is transferred to electrons thereby exciting them to higher energy states into the conduction band, hence, reducing the resistance. Opposite happens when less intensity light falls on the sensor.When the LED used emits light in cloudy water towards the LDR placed in the line of sight, the intensity of light reaching the LDR will be less thereby increasing the resistance compared to the resistance of LDR when light from LED is emitted in clean water. Using this ability of LDR a measurement circuit is built as shown in figure 2. As a result the clean water will result in lower potential compared to the cloudy water.

\section{Microcontroller}

The proposed model for water resource monitoring uses ESP8266 as the microcontroller. ESP8266 supports a WiFi module and is a SoC device. ESP8266 mainly finds applications in IoT embedded systems due to its low cost, processing capability and most importantly the WiFi support. It has a support of $2.4 \mathrm{GHz} \mathrm{WiFi}$ module, 10 bit analog to digital convertor, 16 GPIO pins, Serial Peripheral Interface (SPI), direct memory access, I2C, I2S, UART and supports PWM which is pulse width modulation.

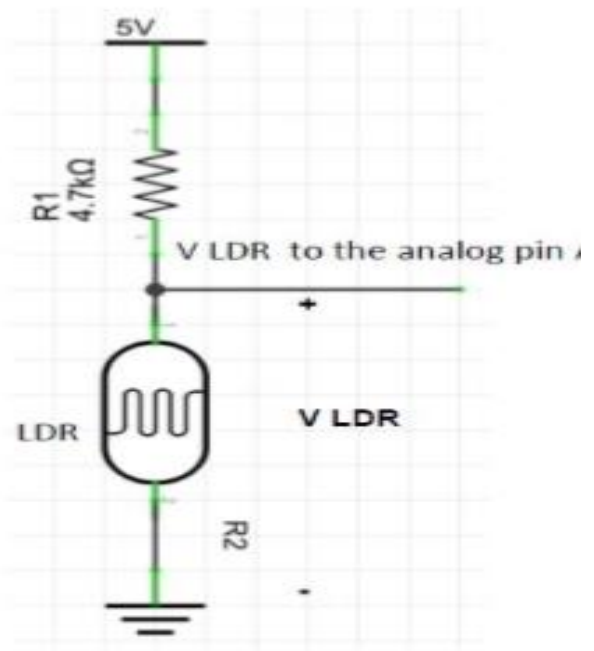

Fig. 2. Voltage divider for sensing LDR

ESP8266 makes use of a 32 bit RISC processing unit which is based on Xtensa L106 operating at $80 \mathrm{MHz}$. It supports 64 $\mathrm{KB}$ bootable read only memory (ROM) along with $96 \mathrm{~KB}$ data RAM and $64 \mathrm{~KB}$ instruction RAM.

Published By:

Blue Eyes Intelligence Engineering

\& Sciences Publication

(C) Copyright: All rights reserved.

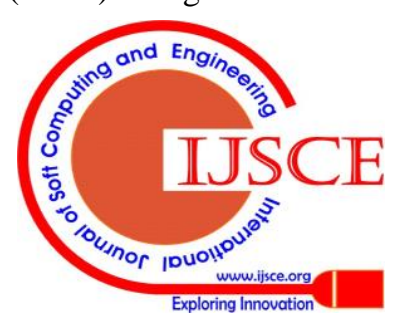




\section{Household Water Resource Monitoring and Leakage Detection}

Role of microcontroller is both to give power supply to sensor devices and collect data from the sensors present in the system for further data processing using machine learning and sensor fusion techniques to monitor water consumption and leakage detection. ESP8266 is used at each branch to collect flow sensor data and is also used in tank to collect sensor data.

\section{Ultrasonic sensor and flow sensor}

Ultrasonic sensor plays an important role in our water monitoring system for determining the level of water in the tank. Ultrasonic sensor is a transducer which uses a circuitry to propagate out ultrasonic sound signal pulses and receive them back which inform about the water level in the tank. These ultrasonic sound pulses are present at high frequency which reflect from boundaries to generate distinct echoes. The distance between the target and the sensor is determined by calculating the time delay between the received pulse and the sent pulse. In the proposed system the ultrasonic sensor is located upright at the closing portion of the tank and is faced towards the water.

Flow sensor is an electronic transducer which is used to determine the rate of flow of water in each branch of the water system. Flow sensors are made up of water rotor, hall effect sensor and is covered by a plastic body. The water flowing through the branches rotate the rotor and the speed of rotation of the rotor depends on the speed of the water flow. The hall effect sensor gives out the associated pulse signal. Data collected from the ultrasonic sensor and flow sensor are used to determine the usage.

\section{METHODOLOGY}

The microcontroller collects data from various flow sensors and ultrasonic sensor. Flow sensors are kept near every tap. Data collected is uploaded to thingspeak cloud. Thingspeak data channels are created for each flow sensor and ultrasonic sensor. The system can be described as follows:

A. Leakage detection

1. The machine learning model assumes that a new water supply network would not have any leakage issues for a definite period of time. Consequently the data collected during that period of time is the training data.

2. A model is trained with the data uploaded to the cloud. Inference is done on the trained model and leakage is detected and reported.

3. A mobile app gives a notification, if there is a leakage.

B. Usage monitoring

1. Values from flow sensors are uploaded to cloud.

2. Water usage is calculated from flow values.

3. Amount of water in terms of height is also notified to the user.

4. If the tank is filled the user can be notified about it. Loss of water can be prevented.

5. If the tank is empty, the user is notified to turn on the motor to fill the tank.

6. All the monitoring and notifications are displayed on a user mobile App.

\section{Water turbidity levels}

1. LED and LDR circuit is used to measure turbidity of water in overhead tank.

2. LDR values are uploaded to thingspeak channel.

3. LDR value above a preset threshold indicates the user to clean the tank.

\section{A. Machine learning model for leakage detection}

Machine learning (ML) plays a very important role in the leakage detection by continuously learning from the data provided to the model. The ML model implemented is based on the idea that the flow rate has a dependency on the height of the water in the tank, hence, the leakage detection has to be carried out based on this dependency. But, it can observed experimentally that at every height most of the flow values are possible and should not be treated as anomaly when considered individually but the difference between the height values for given flow values should not differ significantly because the amount of reduction in the level of water is related to flow values in an ideal scenario but in real scenario sensor values can fluctuate between a small range which will not amount to exact increase/decrease in level.The fluctuation between these values must not be considered as an anomaly for leakage but instead part of the distribution because the sensitivity of the sensor could be high. Sporadically, the sensor values can be highly abnormal and cannot be a part of the distribution, such values are sensor reading anomalies and not the leakage anomaly. Therefore, it is imperative to segregate the sensor value anomaly with actual leakage value anomaly. Furthermore considering the separation of these two anomalies assists in reducing the false positives significantly. So, in order to detect leakage, two models are used, which are given below:

- Model to detect anomalous sensor readings.

- $\quad$ Model to detect leakage.

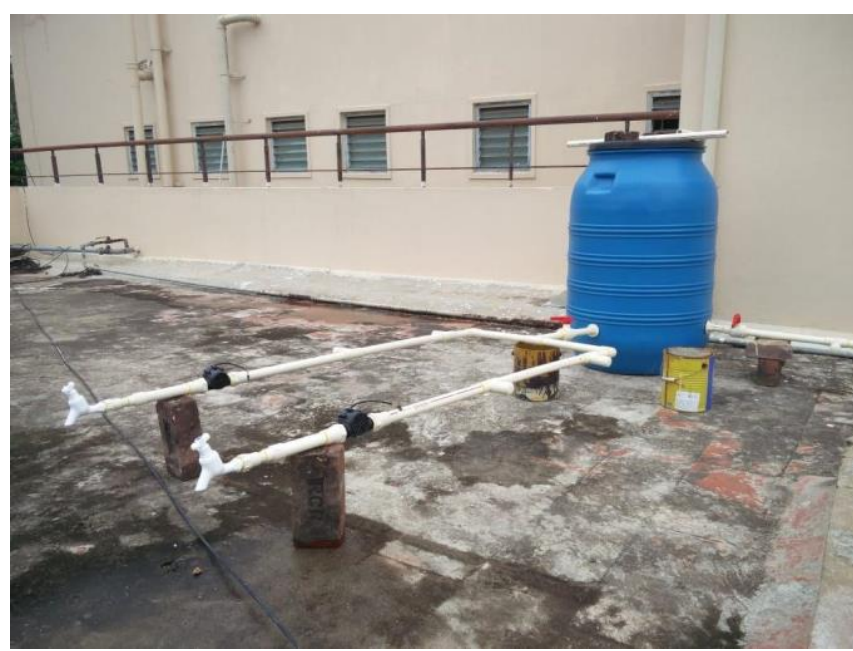

Fig. 3. Prototype of the proposed system

To detect anomalous sensor reading, Isolation Forest algorithm is used to separate the outlier points from the rest of the distribution. Ideally outlier points can be isolated with as few iterations as possible.

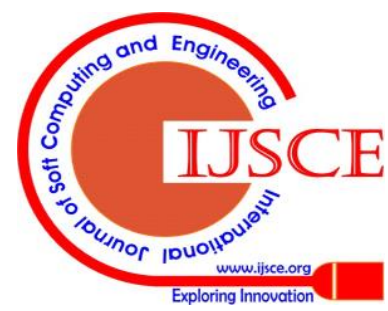




\section{Household Water Resource Monitoring and Leakage Detection}

Once a set of points are classified as non-anomalous then these set of points are sent to second model to detect leakage.

Let $Y 1$ denote the state of sensor values output set and $Y 2$ output of the Leakage model and $Y$ be the state of the entire system (0 for non-anomalous/1 for anomalous/P for previous state of the system). Action is taken only if $Y 1=0$ and at $Y 1=1$ as shown in Table 1 . The other set of points are not considered for leakage model input since the value returned by the sensor is found to be anomalous by the isolation forest model. Therefore these points cannot be considered to evaluate the state of the entire system.

Table 1. Decision table

\begin{tabular}{|l|l|l|}
\hline$Y_{1}$ & $Y_{2}$ & $Y$ \\
\hline 0 & 0 & 0 \\
\hline 0 & 1 & 1 \\
\hline 1 & - & $P$ \\
\hline 1 & - & $P$ \\
\hline
\end{tabular}

The general parameters of the model are $\left\{\mathbf{s}_{1}, \mathbf{s}_{2}, \mathbf{s}_{3}, \mathbf{S}_{4}, \ldots . \mathbf{s}_{\mathbf{n}}\right.$, $\mathbf{H}$ \} where $\mathbf{s}_{\mathbf{f}} \mathbf{f} \mathbf{\epsilon} \mathbf{I}$ are sensor flow rate and $\mathbf{H}$ is the height of the tank. Feature Engineering is done on collected data and extra parameters are added to involve the effect of time on the system.

$\Delta \mathbf{h}_{\mathrm{k}}=\left(\mathbf{h}-\mathbf{h}_{\mathrm{k}}\right)$ for $\mathbf{k}=\left\{\mathbf{h}(\mathbf{i}) \mid \mathbf{t}_{\mathrm{i}}<\mathbf{t}_{\mathrm{k}}\right\}$, where $\mathbf{h}(\mathbf{i})$ denotes height value at $\mathbf{t}_{\mathbf{i}}$ sample and $\mathbf{t}_{\mathbf{i}}$ denotes the ith sample.To detect value of $\mathbf{Y} \mathbf{2}$ and subsequently detect leakage following operations are performed.

1. The system considers overlapping of samples to determine the state of $\mathbf{Y} \mathbf{2}$. Let $\mathbf{T}$ denote the time period where the system checks for leakage then $\Delta t$ be the max of time difference taken to calculate $\Delta \mathbf{h}_{\mathrm{k}}$ that is: $\max \left(\mathbf{t}-\mathbf{t}_{\mathrm{k}}\right) \quad \mathbf{V} \Delta \mathbf{h}_{\mathrm{k}}$ considered as parameters. The condition of $\mathbf{T}<\Delta \mathbf{t}$ must be true for the model to detect leakage.

2. The condition ensures that if the system checks at time $\mathbf{t}$ then certain subset of samples in $[t-\Delta t$, $\mathbf{t}]$ are considered in both $\{\mathbf{t}-\mathbf{T}, \mathbf{t}\}$ that is: If the state of $\mathbf{Y} \mathbf{2}$ is actually $\mathbf{1}$ then it will detected as $\mathbf{1}$ in either $\mathbf{t}-\mathbf{T}$ or in $\mathbf{t}$ and if $\mathbf{Y} \mathbf{2}$ is $\mathbf{0}$ then in both $\mathbf{t}-\mathbf{T}$ and $\mathbf{t}$ the state of $\mathbf{Y} \mathbf{2}$ will be $\mathbf{0}$.

3. K-Means clustering is applied after feature engineering to determine the clusters where points belong. The optimum number of clusters are determined through the Elbow graph method.

4. The centroids of the clusters are identified then a new set of samples for testing collected from $\left[t_{t}-\Delta t, t_{t}\right]$ where $\mathbf{t}_{\mathrm{t}}$ time at which testing is done the values of $\Delta \mathbf{h}_{\mathrm{k}}$ are calculated and the parameter set becomes $\left\{\mathbf{s}_{1}, \mathbf{s}_{2}, \mathbf{s}_{3}\right.$, $\left.\mathrm{S}_{4}, \ldots . \mathrm{S}_{\mathrm{n}}, \mathrm{H}\right\} \mathrm{U}\left\{\Delta \mathrm{h}_{\mathrm{k}}\right\}$.

5. Euclidean distance is used to calculate the distance (D) of the parameter set with the centroid of the clusters. If $\left(\mathbf{D}_{i}>\boldsymbol{\mu}_{i}+f^{*} \boldsymbol{\sigma}_{\mathrm{i}}\right)$ where $\mathbf{i}$ denotes cluster number, $f$ is scalar and positive integer where $f=1$ accounts for $68 \%$ of complete variance in data, similarly $\mathrm{f}=2$ accounts for

Rotripisal Number: F3393039620/2020@BEIESP
$95 \%$ and $\mathrm{f}=3$ for $99.7 \%$ this value of $\mathrm{f}$ can be calculated by multiple trials and be matched with percentage of anomaly, from our case $\mathrm{f}=2$ is found to be optimal, Here $\boldsymbol{\mu}$ is average distance of the points of the corresponding cluster to its centroid and $\boldsymbol{\sigma}$ is standard deviation when this condition holds true then leakage has occurred otherwise the sample collected is within the distribution.

\section{RESULTS AND DISCUSSIONS}

When the system was tested, leakage in the water system is detected when higher values or lower values which are close to 0 are detected as leakage. Tables 2 and 3 below show leakage detected and not detected respectively.

Table 2. Leakage detected

\begin{tabular}{|l|l|l|l|l|l|}
\hline $\begin{array}{c}\text { Height } \\
(\mathrm{H})\end{array}$ & $\begin{array}{c}\text { Sensor1 } \\
(\mathrm{s} 1)\end{array}$ & $\begin{array}{c}\text { Sensor2 } \\
(\mathrm{s} 2)\end{array}$ & \multicolumn{1}{|c|}{$\mathrm{h} 1$} & $\mathrm{~h} 2$ & $\mathrm{~h}$ \\
\hline 38.28 & 0.68 & 0.81 & 1.34 & 2.27 & 2.24 \\
\hline 39.19 & 0.81 & 0.68 & -1.26 & 2.46 & 2.17 \\
\hline 40.22 & 0.68 & 0.81 & -1.46 & 2.08 & -2.26 \\
\hline 40.82 & 0.68 & 0.68 & 1.86 & 2.34 & 2.26 \\
\hline
\end{tabular}

Table 3. Leakage not detected

\begin{tabular}{|l|l|l|l|l|l|}
\hline $\begin{array}{c}\text { Height } \\
(\mathrm{H})\end{array}$ & $\begin{array}{c}\text { Sensor1 } \\
(\mathrm{s} 1)\end{array}$ & $\begin{array}{c}\text { Sensor2 } \\
(\mathrm{s} 2)\end{array}$ & \multicolumn{1}{|c|}{ h1 } & \multicolumn{1}{|c|}{ h2 } & h3 \\
\hline 43.36 & 0.68 & 0.68 & 0.61 & 0.55 & -0.34 \\
\hline 43.46 & 0.4 & 0.68 & 0.1 & 0.71 & 0.65 \\
\hline 43.56 & 0.4 & 0.68 & 0.1 & 0.2 & 0.81 \\
\hline 43.73 & 0.27 & 0.54 & 0.17 & 0.27 & 0.37 \\
\hline
\end{tabular}

Figure 4 and figure 5 shows the height of the water in the tank and flow sensor values at two branches in the mimicked model as shown in figure 3 . In figure 4 we can see that the relationship between height and flow values is linear. Figure 5 indicates the values of flow sensors are two different branches with respect to time. The pattern of flow sensor values can be observed.The taps are rotated in this manner so that the training the model is easier.
Published By:

Blue Eyes Intelligence Engineering \& Sciences Publication (C) Copyright: All rights reserved.

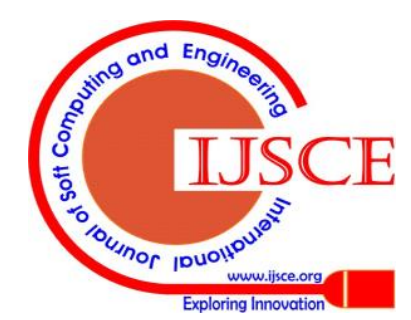




\section{Household Water Resource Monitoring and Leakage Detection}

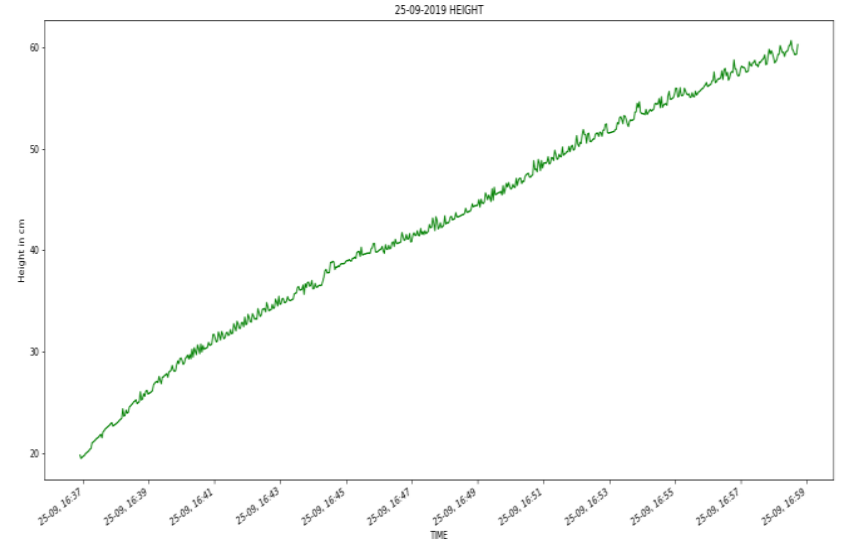

Fig. 4. Height of water in the tank vs time

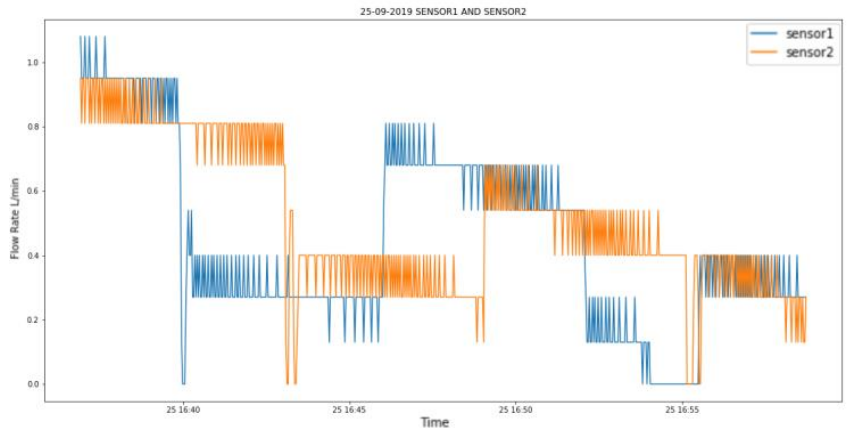

Fig. 5. Flow sensor values at different instants connected at 2 branches of the water system. (Flow rate vs Time)

Figure 6 shows the anomaly graph, which can be used to differentiate the anomalous sensor readings. Anomalous sensor readings are first removed before giving it to the model. Sensor values which deviate too much from the normal readings are discarded. The blue dots in the graph indicate the anomalous readings obtained from the isolation forest algorithm. Such outlier points are removed from the distribution.

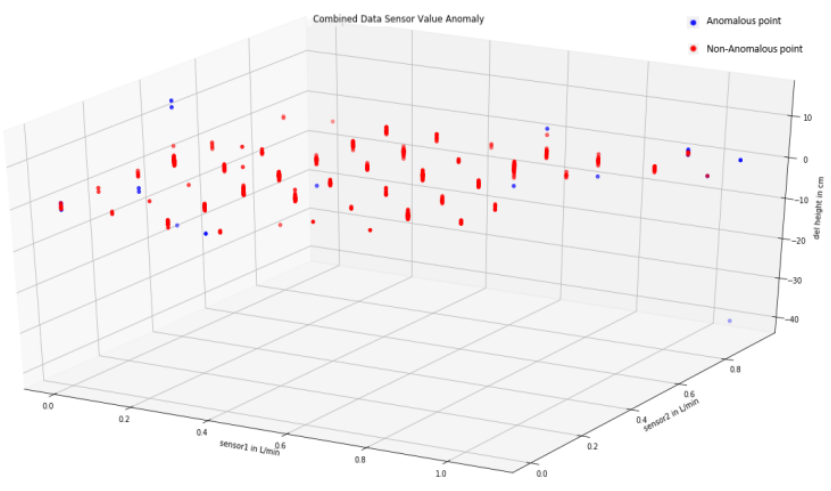

Fig. 6. Sensor anomaly graph

\section{CONCLUSION AND FUTURE SCOPE}

In the paper a real time, low cost, energy efficient water resource monitoring and leakage detection system is presented. The proposed system efficiently monitors the water usage in households, detects leakage and predicts the future usage using machine learning and sensor fusion techniques and notifies the user about these parameters. Most importantly, the current global demand and environmental conditions have made it necessary to conserve water which involves creating innovative solutions and implementations. So, the proposed system can be effectively implemented in houses for continuously monitoring the usage and detection of leakage if any and notifying the people about the same, helping users to control their usage and immediately take action if any anomaly in the water system is detected.

Future works include using of multiple water quality sensors to monitor water quality and incorporating the entire water monitoring system with a much larger environment monitoring system (which includes monitoring all the surrounding parameters like air, water and energy).

\section{REFERENCES}

1. A. M. Manoharan and V. Rathinasabapathy, "Smart Water quality Monitoring and Metering Using Lora for Smart Villages", 2018 2nd Internationalconference on Smart Grid and Smart Cities (ICSGSC), Kuala Lumpur, 2018, pp. 57-61.

2. N. R. Moparthi, C. Mukesh and P. Vidya Sagar, "Water Quality Monitoring System Using IoT", 2018 Fourth International Conference on Advances in Electrical, Electronics, Information, Communication and Bioinformatics (AEEICB), Chennai, 2018, pp. 1-5.

3. G. S. Menon, M. V. Ramesh and P. Divya, "A low cost wireless sensor network for water quality monitoring in natural water", 2017 IEEE Global Humanitarian Technology Conference (GHTC), San Jose, CA, 2017, pp. 1-8.

4. C. Sowmya, C. D. Naidu, R. P. Somineni and D. R. Reddy, "Implementation of Wireless Sensor Network for Real Time Overhead Tank Water Quality Monitoring", 2017 IEEE 7th International Advance Computing Conference (IACC), Hyderabad, 2017, pp. 546-551.

5. Abhinav S; Sahana S; Aishwarya G; Anala M R; Mamatha T, "Wireless Water Quality Monitoring and Quality Deterioration Prediction System," 2019 26th International Conference on High Performance Computing, Data and Analytics Workshop (HiPCW), 2019, pp. 23-28.

6. K. Indu and J. J. Choondal, "Modeling development and analysis of low cost device for water quality testing", 2016 IEEE Annual India Conference (INDICON), Bangalore, 2016, pp. 1-6.

7. S. N. Ibrahim, M. S. L. Hakim, A. L. Asnawi and N. A. Malik, “Automated Water Tank Filtration System Using LDR Sensor", 2016 International Conference on Computer and Communication Engineering (ICCCE), Kuala Lumpur, 206, pp. 195-199.

8. T. Perumal, M. N. Sulaiman and C. Y. Leong, "Internet of Things (IoT) enabled water quality monitoring system", 2015 IEEE 4th Global Conference on Consumer Electronics (GCCE), Osaka, 2015, pp. 86-87.

\section{AUTHORS PROFILE}

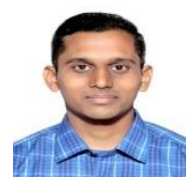

Salman Asif $\mathbf{S}$ is a student at R.V College of Engineering.he is a B.E student, currently studying in 8th sem ,computer science domain. His area of interest includes machine learning.

Sachin R Doddaguni is a student .at R.V College of Engineering.he is a B.E student, currently studying in 8th sem ,computer science domain.His area of interest includes applications of cloud computing and machine learning.

Published By:

Blue Eyes Intelligence Engineering DOI:10.35940/ijsce.F3393.059620

Journal Website: www.ijsce.org

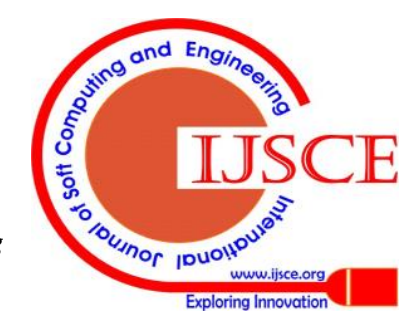




\section{Household Water Resource Monitoring and Leakage Detection}

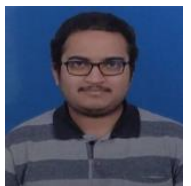

Trilok Girish Kamagond is a student at RV College Of Engineering. He is a B.E. student, currently studying in 8th sem, electronics and communication domain. His interests are in sensors, analog and machine learning.

Dr. Anala M. R., is an Associate Professor at R.V College of Engineering. She has over 18 years of experience in teaching. Main area of research interest is Computer Architecture, High Performance Computing, Distributed Systems and Parallel programming. She has guided 40 UG projects and 20 PG projects. She has many publications in international journals and conferences.

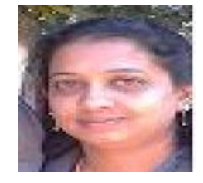

Prof. Mamatha $\mathbf{T}$ is having Masters Degree in Computer Science and Engineering and pursuing her $\mathrm{PhD}$ in the area of Internet of Vehicles. She has wide experience of 19 years in teaching and research. She is working as Assistant Professor at Department of Computer Science \& Engineering at RV College of Engineering. The author has good research publications. Her research interest includes Embedded Systems, D2D communication, Internet of Vehicles. 